\section{PTH-132 UK MEDICAL STUDENT PERCEPTIONS ON DIET AND NUTRITION EDUCATION: RESULTS OF A NATIONAL SURVEY}

Dominic Crocombe*. Barts And The London Medical School, London, UK

10.1136/gutjnl-2018-BSGAbstracts.531

Introduction Dietary modification is a fundamental component of preventing and managing many gastrointestinal conditions and lifestyle-related diseases. Questions about diet are often the primary concern of patients and great attention is drawn to this field by scientists and non-scientists in popular and social media. This national survey explored UK medical students' views on the responsibility of doctors, confidence in their current knowledge and ability to give advice, and the perceived quality of their medical school education regarding diet and nutrition in health and disease.

Methods An online survey of Likert-type questions and freetext spaces was distributed to medical student unions and medical school societies of UK medical schools via email and social media platforms.

Results 181 (91 pre-clinical, 90 clinical) students from 11 UK medical schools completed the survey. 96\% felt doctors have a responsibility to provide the best available evidence-based dietary advice to patients. Overall, 63\% reported being asked about diet and nutrition by patients 'occasionally' or 'often'. $40 \%$ of students had confidence in their current knowledge and $32 \%$ did not. Confidence was generally low (21\% confident, 55\% not) regarding knowledge of UK dietary guidelines. Awareness of many popular dietary philosophies that patients might enquire about - such as the Mediterranean, intermittent fasting and low-carb-high-fat diets - was high but students did not feel confident in advising patients regarding these. Only $14 \%$ of students (16\% of pre-clinical, $10 \%$ of clinical), felt they were receiving a comprehensive and relevant education in diet and nutrition from their medical school [Graph 1]. Over two-thirds $(68 \%)$ would appreciate more teaching in this area and $25 \%$ had already sought further education themselves.

Conclusions There is some feeling amongst medical students that UK medical schools are failing to provide an adequate education in clinical nutrition and dietetics. This is despite a desire for better education in this area and a widespread belief that doctors have a responsibility to impart high quality dietary advice to their patients. Medical student confidence in their own knowledge varied but was generally low regarding official UK guidelines and some of the more popular dietary philosophies that patients may enquire about. The main limitations of this study are its small sample size and risk of sampling bias. Medical school faculties might consider canvassing their students with a view to improving their teaching on diet and clinical nutrition.

\section{PTH-133 FIVE YEARS OF HIFIVE (HUMAN FACTORS IN VIRTUAL ENDOSCOPY): AN ENDOSCOPIC NON-TECHNICAL SKILLS SIMULATION PROGRAMME}

Tareq El Menabawey*, Valerie Dimmock, Radha Gadock, Sam Murray, Vathsan Ravindran, Eleanor Wood. Homerton University Hospital, London, UK

\subsection{6/gutjnl-2018-BSGAbstracts.532}

Introduction Endoscopic non-technical skills (ENTS) are increasingly recognised as a vital component of endoscopic ability. The
Joint Advisory Group on GI endoscopy (JAG) have included them in their updated direct observation of procedural skills (DOPS) assessments of endoscopy competency and therefore competence in these skills is required for accreditation. However, a formal framework for teaching these skills has not yet been established. Here we describe our centre's five year experience of running a one-day multidisciplinary immersive simulation course aimed at developing ENTS matched to the JAG curriculum.

Methods From 2012-17, we have run 8 simulation days involving 5 standardised scenarios based on real life serious incidents, using simulated patients, an endoscopy simulator (GI Mentor Express Symbionix) and vital signs consoles (iSimulate). Doctors, nurses and healthcare assistants from endoscopy units attended. From 2012-16 post course questionnaires were completed by attendees focussed on learners' perception of usefulness of the course on a 4 point Likert scale. From 2016-17 these questionnaires were updated to include pre and post course confidence scores in eight domains reflecting ENTS scored on a 3 point Likert scale. Student's t-test was used to calculate statistical significance between pre and post scores in Microsoft Excel. Qualitative statements on what attendees had learned and how it would impact practice were also collected.

Results We retrospectively analysed the questionnaire responses of 22 doctors, 21 nurses and two healthcare assistants. From 2012-16 weighted averages of responses across all 21 statements indicate acceptability of simulation as a technique for teaching ENTS (median number of respondents $=38$ ).

From 2016-17 the average confidence across all eight domains increased (median number of respondents $=16$ ). Three of these achieved statistical significance $(p<0.01)$ - 'I can manage an emergency clinical care scenario using time and resources efficiently', 'I can manage complaints effectively with positive outcomes' and 'I can understand the human factors that play a role in complex clinical care situations' (Chart 1).

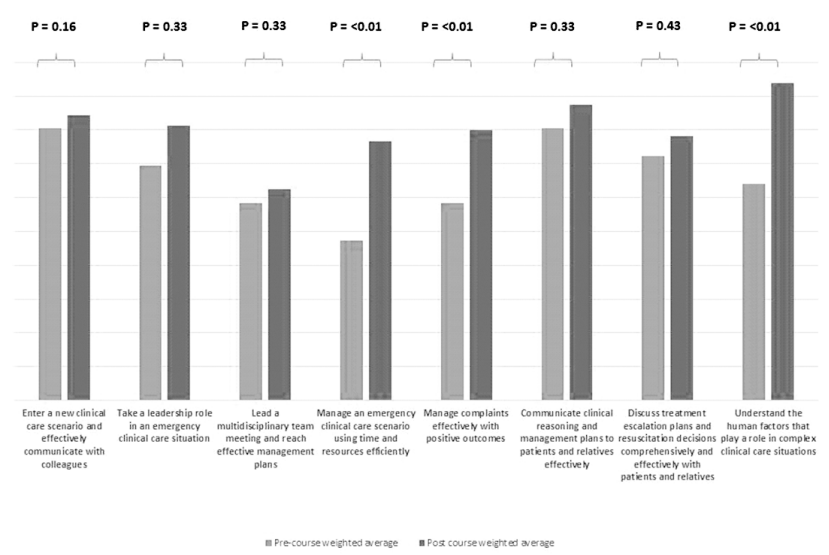

Abstract PTH-133 Figure 1

Qualitative statements of lessons learned indicated that attendees found debriefing with the actor giving a patient perspective a very informative experience, increasing patient centeredness and discovering useful strategies for future practice. Conclusions Over the last 5 years, this course has attracted attendees within our own deanery and nationally. We have shown that a one-day ENTS simulation course is feasible and can have a significant impact on trainees' confidence in their non-technical skills. We believe this is a good model for delivering ENTS training for other centres to follow. 